\title{
High-Order Hybridizable Discontinuous Galerkin Method for the Gas Kinetic Equation
}

\author{
Wei Su, Peng Wang and Yonghao Zhang \\ James Weir Fluids Laboratory, Department of Mechanical and Aerospace Engineering, \\ University of Strathclyde, G1 1XJ Glasgow, United Kingdom
}

\author{
ARTICLE HISTORY \\ Compiled July 31, 2019
}

\begin{abstract}
The high-order hybridizable discontinuous Galerkin (HDG) method is used to find the steady-state solution of gas kinetic equation on two-dimensional geometry. The velocity distribution function and its traces are approximated in the piecewise polynomial space on triangular mesh and mesh skeleton, respectively. By employing a numerical flux that is derived from the upwind scheme and imposing its continuity on mesh skeleton, the global system for unknown traces is obtained with fewer coupled degrees of freedom, compared to the original DG method. The steady-state solution is reached through a semi-implicit iterative scheme. The solutions of linearized model equation for the Poiseuille flow through square channel show that the higher-order solver is faster than the lower-order one. Moreover, the HDG scheme is more efficient than the original DG method when the degree of approximating polynomial is larger than 2. Finally, the developed scheme is extended to solve the Boltzmann equation with full collision operator, which can produce accurate results for shear-driven and thermally induced flows.
\end{abstract}

\section{KEYWORDS}

hybridizable DG, Boltzmann equation, rarefied gas flow, upwind flux

\section{Introduction}

Gas flow widely encountered in high-altitude aerodynamics, vacuum technology, shale gas extraction and micro-electro-mechanical system (MEMS), is often beyond the continuum flow regime, where the evolution of macroscopic flow properties such as density, bulk velocity and temperature is no longer governed by the conventional Navier-Stokes (NS) equations. To predict such rarefied gas flows, microscopic description for the motion of gas molecules is necessary. In gas kinetic theory, the system state is described by the velocity distribution function (VDF) $f$. It is a function of time $t$, position $\boldsymbol{x}$ and molecular velocity $\boldsymbol{v}$, and is defined such that $f \mathrm{~d} \boldsymbol{x} \mathrm{d} \boldsymbol{v}$ is the number of gas molecules in the phase-space (physical space and velocity space) volume $\mathrm{d} \boldsymbol{x} \mathrm{d} \boldsymbol{v}$. The macroscopic flow properties are derived via velocity moments of VDF. The evolution of $f$ is governed by the Boltzmann equation (Chapman and Cowling 1970)

$$
\frac{\partial f}{\partial t}+\boldsymbol{v} \cdot \frac{\partial f}{\partial \boldsymbol{x}}+\boldsymbol{a} \cdot \frac{\partial f}{\partial \boldsymbol{v}}=\mathcal{C}, \quad \mathcal{C}=\iint B \cdot\left(f_{*}^{\prime} f^{\prime}-f_{*} f\right) \mathrm{d} \Omega \mathrm{d} \boldsymbol{v}_{*}
$$

CONTACT Wei Su. Email: wei.su@strath.ac.uk 
where, $\boldsymbol{a}$ is the external force exerting on a volume of unit mass of gas molecules and $\mathcal{C}$ is the collision operator that describes change in VDF due to binary collisions. Nomenclature for other arguments is referred to (Wu et al. 2013). The multidimensional nature (i.e. six dimensions in the phase space and one dimension in the temporal space) of the Boltzmann equation poses a real challenge to the numerical solution. Two categories of numerical approaches have been developed. One is the stochastic method that uses simulating particles to mimic the molecular behavior and the other is the deterministic method that relies on the discretization of governing equation over computational grid (Dimarco and Pareschi 2014). The deterministic approaches are based on the discrete velocity model, which uses a set of $M_{\mathrm{v}}$ discrete $\boldsymbol{v}^{j}$ to discretize the molecular velocity space, resulting in a system of equations evaluated at each $\boldsymbol{v}^{j}$

$$
\frac{\partial f^{j}}{\partial t}+\boldsymbol{v}^{j} \cdot \frac{\partial f^{j}}{\partial \boldsymbol{x}}+\boldsymbol{a} \cdot\left(\frac{\partial f}{\partial \boldsymbol{v}}\right)_{j}=\mathcal{C}^{j}, \quad j=1, \ldots, M_{\mathrm{v}},
$$

where $f^{j}(t, \boldsymbol{x})=f\left(t, \boldsymbol{x}, \boldsymbol{v}^{j}\right)$. Then, the equations that are still continuous in the physical space and time are solved by computational fluid dynamic (CFD) technologies. Usually, a large number, e.g. several ten thousands, of discrete velocities (discrete equations) are required to accurately capture the variation of VDF. This makes the computational cost immediately prohibitive for realistic problems. Thus, high-order CFD method is critical to reduce the number of degree of freedom (DoF).

One of the promising methods for this purpose is the discontinuous Galerkin (DG) method, which was first introduced for the neutron transport equation (Reed and Hill 1973). After combing an explicit Runge-Kutta (RK) time marching scheme, the method has great success in solving convection-dominated problems (Cockburn and Shu 2001). In recent years, the explicit DG method has been applied to solve the gas kinetic equations (Gobbert, Webster, and Cale 2007; Evans, Morgan, and Hassan 2011; Kitzler and Schöberl 2015). It was shown that the $2^{\text {nd }}$-order DG method is 15 times more efficient than the $2^{\text {nd }}$-order finite volume scheme in solving the kinetic model equations (Su, Alexeenko, and Cai 2015). Another attractive feature is that DG can naturally obtain fluxes at the boundaries with the same high-order accuracy as in the interior of the domain (Mieussens 2014). This is particularly important for solution of flow in micro-systems, where the surface-to-volume ratio is significantly magnified.

Despite the above advantages, it was shown that higher-order RKDG scheme is not superior to lower-order one, e.g. the $3^{\text {rd }}$-order scheme is about 4 times slower than the $2^{\text {nd }}$-order one to obtain solutions with the same order of accuracy for rarefied Couette flow (Su, Alexeenko, and Cai 2015). It is mainly due to the two facts for explicit high-order scheme: 1) number of DoF rapidly increases; 2) time step restricted by the Courant-Friedrichs-Lewy (CFL) condition becomes extremely small (Kubatko, Dawson, and Westerink 2008), thus number of iteration becomes larger in finding steady-state solution. Although implicit scheme could be used to relax the CFL restriction, the classical DG methods are computationally expensive for implicit gas kinetic solvers, since the number of globally coupled DoF is significantly large. The hybridizable discontinuous Galerkin (HDG) method was proposed to overcome this disadvantage (Cockburn, Gopalakrishnan, and Lazarov 2009). By producing a final system in terms of DoF in approximating the traces of field variables, HDG could significantly reduce the number of global unknowns, since the traces are defined on cell interfaces and single-valued. The majority of HDG applications in fluid dynamics to date includes convection-diffusion flow (Cockburn, Gopalakrishnan, 
and Lazarov 2009), Stokes flow (Nguyen, Peraire, and Cockburn 2010), wave propagation problem (Giorgiani, Fernández-Méndez, and Huerta||2013) and incompressible/compressible NS flows (Peraire, Nguyen, and Cockburn 2010). In this paper, we present a HDG scheme that is designed for the gas kinetic equation for the first time.

\section{HDG formulation}

We present the HDG formulation to the linearized BGK model equation (Bhatnagar, Gross, and Krook 1954). When the flow velocity and the external acceleration are sufficiently small, we can linearize VDF about the global equilibrium state $f_{\text {eq }}=$ $\exp \left(-|\boldsymbol{v}|^{2}\right) / \pi^{3 / 2}$ as $f=f_{\text {eq }}(1+h)$, where $h$ is the perturbed VDF. Then, interpreting $f$ by $h$ in Eq. (1) and neglecting the nonlinear terms, we have the following equations for $h^{j}$ (Cercignani 1988)

$$
\boldsymbol{v}^{j} \cdot \frac{\partial h^{j}}{\partial \boldsymbol{x}}-2 \boldsymbol{a} \cdot \boldsymbol{v}^{j}=\frac{\sqrt{\pi}}{2 K n}\left(\mathcal{L}^{j}-h^{j}\right), \quad \mathcal{L}^{j}=\varrho+2 \boldsymbol{u} \cdot \boldsymbol{v}^{j}+\tau\left(\left|\boldsymbol{v}^{j}\right|^{2}-\frac{3}{2}\right),
$$

where $\varrho$ is the perturbed density, $\boldsymbol{u}$ is the bulk velocity and $\tau$ is the perturbed temperature. $\mathcal{L}^{j}$ is the linearized equilibrium distribution. $K n$ is the Knudsen number related to the characteristic flow dimension $H$ and the gas properties (pressure $p_{0}$, temperature $T_{0}$ and viscosity $\mu_{0}$ ) at reference condition as $K n=\mu_{0} \sqrt{\pi R T_{0}} / \sqrt{2} p_{0} H$, where $R$ is the specific gas constant. We have omitted the derivative with respect to time in Eq. (3) since we are only interested in steady-state solutions. Macroscopic properties are calculated through quadratures

$$
\varrho=\sum_{j=1}^{M_{\mathrm{v}}} h^{j} f_{\mathrm{eq}}^{j} \varpi^{j}, \quad \boldsymbol{u}=\sum_{j=1}^{M_{\mathrm{v}}} \boldsymbol{v}^{j} h^{j} f_{\mathrm{eq}}^{j} \varpi^{j}, \quad \tau=\frac{2}{3} \sum_{j=1}^{M_{\mathrm{v}}}\left|\boldsymbol{v}^{j}\right|^{2} h^{j} f_{\mathrm{eq}}^{j} \varpi^{j}-\varrho,
$$

where $\varpi^{j}$ is the weight of the quadrature rule. All variables are given in dimensionless form: $\boldsymbol{x}$ is normalized by $H ; \boldsymbol{v}$ and $\boldsymbol{u}$ are normalized by the most probable molecular speed $v_{\mathrm{m}}=\sqrt{2 R T_{0}} ; T$ is normalized by $T_{0} ; \mathrm{VDF}$ is normalized by $n_{0} / v_{\mathrm{m}}^{3}$. Note that $\mathcal{L}^{j}$ contains macroscopic variables. To decouple the equations, Eqs. (3) are usually solved by the following semi-implicit iterative scheme

$$
\frac{\sqrt{\pi} h^{j,(t+1)}}{2 K n}+\boldsymbol{v}^{j} \cdot \frac{\partial h^{j,(t+1)}}{\partial \boldsymbol{x}}=\frac{\sqrt{\pi}}{2 K n} \mathcal{L}^{j,(t)}+2 \boldsymbol{a} \cdot \boldsymbol{v}^{j},
$$

where the superscripts $(t)$ and $(t+1)$ represent two consecutive iteration steps. The iteration is terminated when the convergence to steady solution is achieved. For conciseness, we will omit the step index in the remainder of the paper unless necessary.

\subsection{Weak formulation}

Let $\Delta \in \mathbb{R}^{2}$ be a two-dimensional domain with boundary $\partial \Delta$ in the physical space, which is partitioned into $M_{\mathrm{el}}$ disjoint regular triangles $\left\{\Delta_{i}: \Delta=\cup_{i}^{M_{\mathrm{el}}} \Delta_{i}\right\}$. The boundaries of $\Delta_{i}$ (denoting as $\partial \Delta_{i}$ ) define a group of $M_{\mathrm{fc}}$ faces $\left\{\Gamma_{c}: \Gamma=\cup_{i}^{M_{\mathrm{el}}} \partial \Delta_{i}=\cup_{c}^{M_{\mathrm{fc}}} \Gamma_{c}\right\}$. 
The HDG method provides an approximate solution to $h^{j}$ on $\Delta_{i}$ as well as an approximation to its trace $\hat{h}^{j}$ on $\Gamma_{c}$ in some piecewise finite element spaces $\mathcal{V} \times \mathcal{W}$ of the following forms

$$
\mathcal{V}=\left\{\varphi:\left.\varphi\right|_{\Delta_{i}} \in \mathcal{P}^{k}\left(\Delta_{i}\right), \forall \Delta_{i} \subset \Delta\right\}, \quad \mathcal{W}=\left\{\psi:\left.\psi\right|_{\Gamma_{c}} \in \mathcal{P}^{k}\left(\Gamma_{c}\right), \forall \Gamma_{c} \subset \Gamma\right\},
$$

where $\mathcal{P}^{k}(D)$ denotes the space of $k$-th order polynomials on the domain $D$. Before describing the HDG formulation, we first introduce a collection of index mapping functions (Kirby, Sherwin, and Cockburn 2012) that relate the local edge of a triangle, namely $\partial \Delta_{i}^{e}$ to a global face $\Gamma_{c}$. Since the $e$-th edge of the triangle $\partial \Delta_{i}$ is the $c$-th face $\Gamma_{c}$, we set $\sigma(i, e)=c$ so that $\partial \Delta_{i}^{e}=\Gamma_{\sigma(i, e)}$. Similarly, since the interior face $\Gamma_{c} \in \Gamma \backslash \partial \Delta$ is the intersection of the two triangles, namely left triangle $\Delta_{i^{-}}$and right triangle $\Delta_{i^{+}}$, we set $\eta(c,+)=i^{+}$and $\eta(c,-)=i^{-}$, then we denote $\Gamma_{c}=\partial \Delta_{\eta(c,+)} \cap \partial \Delta_{\eta(c,-)}$. At the boundary face $\Gamma_{c} \in \partial \Delta$, say, only the right triangle is involved.

The HDG method solves problem in two steps (Cockburn, Gopalakrishnan, and Lazarov 2009). First, a global problem is set up to determine the trace $\hat{h}^{j}$ on $\Gamma$. Then, a local problem with $\hat{h}^{j}$ as boundary condition on $\partial \Delta_{i}$ is solved element-by-element to obtain the solutions of $h^{j}$. Introducing $(\cdot)$ and $\langle\cdot\rangle$ as $(a, b)_{D}=\int_{D \subset \mathbb{R}^{2}}(a \cdot b) \mathrm{d} x_{1} \mathrm{~d} x_{2}$ and $\langle a, b\rangle_{D}=\int_{D \subset \mathbb{R}^{1}}(a \cdot b) \mathrm{d} \Gamma$, respectively, the weak formulation of local problem is

$$
-\left(\nabla \varphi, \boldsymbol{v}^{j} h^{j}\right)_{\Delta_{i}}+\sum_{e=1}^{3}\langle\varphi, \hat{\boldsymbol{F}} \cdot \boldsymbol{n}\rangle_{\partial \Delta_{i}^{e}}+\frac{\sqrt{\pi}}{2 K n}\left(\varphi, h^{j}\right)_{\Delta_{i}}=\left(\varphi, s^{j}\right)_{\Delta_{i}}, \quad \text { for all } \varphi \in \mathcal{V}
$$

where $\boldsymbol{n}$ is the outward unit normal vector and $s^{j}=\sqrt{\pi} \mathcal{L}^{j} / 2 K n-2 \boldsymbol{a} \cdot \boldsymbol{v}^{j} . \hat{\boldsymbol{F}}$ is the numerical flux, of which the definition will be given in the following section.

The global problem, used to determine $\hat{h}^{j}$, is obtained by imposing the continuity of normal fluxes at cell interfaces. The weak formulation is:

$$
\left\langle\psi, \hat{\boldsymbol{F}} \cdot \boldsymbol{n}_{\eta(c,+)}\right\rangle_{\Gamma_{c}}+\left\langle\psi, \hat{\boldsymbol{F}} \cdot \boldsymbol{n}_{\eta(c,-)}\right\rangle_{\Gamma_{c}}=0, \quad \text { for all } \psi \in \mathcal{W},
$$

where $\hat{\boldsymbol{F}} \cdot \boldsymbol{n}_{\eta(c,+)}$ and $\hat{\boldsymbol{F}} \cdot \boldsymbol{n}_{\eta(c,-)}$ denote the numerical fluxes calculated from the left and right triangles, respectively. At the boundary faces, $\hat{\boldsymbol{F}} \cdot \boldsymbol{n}_{\eta(c,-)}$ should be replaced by $\hat{\boldsymbol{G}} \cdot \boldsymbol{n}$, i.e. fluxes flowing into the computational domain. Eliminating the field unknowns $h^{j}$ in Eq. (8) with Eq. (7), and assembling over all the faces, the global problem becomes

$$
\mathbb{K}^{j} \hat{\mathbf{H}}^{j}=\mathbb{R}^{j}
$$

where $\hat{\mathbf{H}}^{j}$ is the vector of DoF of $\hat{h}^{j}$ on all the faces. $\mathbb{K}^{j}$ is the global matrix of the linear system, which is highly sparse, since only face unknowns that involve in two adjacent triangles are coupled at each row. Compared to the standard linear DG system, the trace system is much smaller and sparser when $k>1$ (Huerta et al. 2013). Once the values of $\hat{h}^{j}$ are obtained, an element-by-element reconstruction of $h^{j}$ is implemented according to Eq. (7).

Note that the basic HDG formulation is not limited to the linearized BGK equation. It is straightforward to be extended to the nonlinear Boltzmann equation, where the unkowns in Eq. (7) are the full VDF $f$ and its trace $\hat{f}$ instead. Besides, the 
terms $\sqrt{\pi}(\varphi, h)_{\Delta_{i}} / 2 K n$ and $(\varphi, s)_{\Delta_{i}}$ are replaced by $\left(\varphi, \iint B f_{*} \mathrm{~d} \Omega \mathrm{d} \boldsymbol{v}_{*} \cdot f\right)_{\Delta_{i}}$ and $\left(\varphi, \iint B f_{*}^{\prime} f^{\prime} \mathrm{d} \Omega \mathrm{d} \boldsymbol{v}_{*}\right)_{\Delta_{i}}-(\varphi, \boldsymbol{a} \cdot \partial f / \partial \boldsymbol{v})_{\Delta_{i}}$ respectively. The fivefold integral $\iint \cdot \mathrm{d} \Omega \mathrm{d} \boldsymbol{v}_{*}$ and the derivative $\partial / \partial \boldsymbol{v}$ can be calculated by the fast spetral method (Wu et al.|2013). The detailed HDG formulations are listed in the Appendix.

\subsection{Numerical flux and implementation of boundary condition}

In this paper, we define the flux as (take the linearized BGK equation as an example)

$$
\hat{\boldsymbol{F}}^{j} \cdot \boldsymbol{n}=\boldsymbol{v}^{j} \cdot \boldsymbol{n} \hat{h}^{j}+\left|\boldsymbol{v}^{j} \cdot \boldsymbol{n}\right|\left(h^{j}-\hat{h}^{j}\right) .
$$

If inserting the expression into the continuity equation (8) at interior faces, we immediately obtain $\left\langle\psi, \hat{h}^{j}\right\rangle=\frac{1}{2}\left\langle\psi, h_{\eta(c,+)}^{j}+h_{\eta(c,-)}^{j}\right\rangle$. That is, the trace $\hat{h}^{j}$ at the interior face is equal (in a weak sense) to the average of $h_{\eta(c,+)}^{j}$ and $h_{\eta(c,-)}^{j}$, which are the field unknowns evaluated at the interface from the left and right triangles, respectively. Then we have an equivalent expression for $\hat{\boldsymbol{F}} \cdot \boldsymbol{n}$ :

$$
\hat{\boldsymbol{F}} \cdot \boldsymbol{n}_{\eta(c, \pm)}=\left\{\begin{array}{ll}
\boldsymbol{v}^{j} \cdot \boldsymbol{n}_{\eta(c, \pm)} h_{\eta(c, \pm)}^{j}, & \boldsymbol{v}^{j} \cdot \boldsymbol{n}_{\eta(c, \pm)} \geq 0 \\
\boldsymbol{v}^{j} \cdot \boldsymbol{n}_{\eta(c, \pm)} h_{\eta(c, \mp)}^{j}, & \boldsymbol{v}^{j} \cdot \boldsymbol{n}_{\eta(c, \pm)}<0
\end{array},\right.
$$

which means, the definition (10) can recover the first-order upwind principle. To be consistent with the evaluation of fluxes at interior faces, we calculate the boundary flux as $\hat{\boldsymbol{G}} \cdot \boldsymbol{n}=\boldsymbol{v}^{j} \cdot \boldsymbol{n} \hat{h}^{j}+\left|\boldsymbol{v}^{j} \cdot \boldsymbol{n}\right|\left(g^{j}-\hat{h}^{j}\right)$, where $g^{j}$ is the boundary value of $h^{j}$ and $\boldsymbol{n}$ is the outward unit normal vector at the boundary pointing into the flow field.

\section{Results and discussions}

For verification, we first consider the steady gas flow along a channel of square crosssection in the $x_{1}-x_{2}$ plane, subject to a small constant acceleration $a_{3}=0.5$ in the $x_{3}$ direction, where $\left(x_{1}, x_{2}, x_{3}\right)$ are the Cartesian coordinates in the physical space. It is assumed that the channel length is significantly larger than the dimension of its crosssection, thus the end effect can be neglected and the flow field only varies in the $x_{1}$ and $x_{2}$ directions. The HDG method of $k$ up to 4 is applied to the linearized BGK equation. It is assumed that gas molecules undergo diffuse reflection on the surface and perturbed VDF at boundary is always zero for this problem. The convergence criterion for the iteration (5) is that the increment in flow velocity between two successive iteration steps $\left|\int u_{3}^{(t+1)}-u_{3}^{(t)} \mathrm{d} \Delta\right| /\left|\int u_{3}^{(t)} \mathrm{d} \Delta\right|$ is less than $10^{-5}$. We call the routines in Intel Math Kernel Library (MKL) to invert matrix, and the Intel MKL PARDISO (Schenk and Gärtner 2004) package to solve the global linear system. The test is done in double precision on single Intel Xeon-E5-2680 processor. The velocity grid is fixed with 24 nonuniform points distributing within a truncation of $[-4,4]$ in each direction (Su et al. 2017). Further refinement of the velocity grid would only improve the solutions by a magnitude no more than $0.5 \%$. The flow is resolved on a domain of $\Delta=[0,1] \times[0,1]$, which is partitioned with uniform triangles, as shown in Fig. 1(a), The typical flow velocity contours obtained by the HDG with $k=4$ at $K n=0.018,0.089$ and 0.89 are 


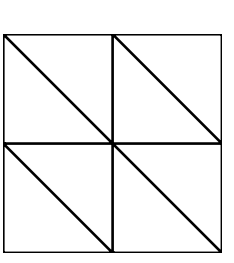

(a) mesh

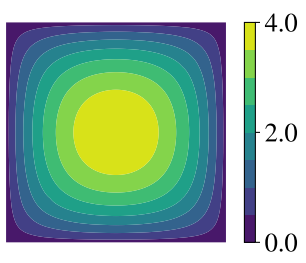

(b) $u_{3}$ at $K n=0.018$

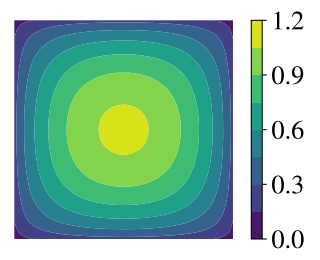

(c) $u_{3}$ at $K n=0.089$

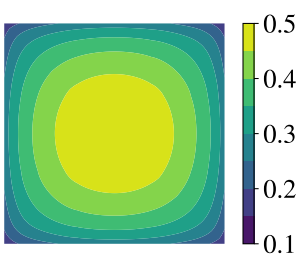

(d) $u_{3}$ at $K n=0.89$

Figure 1. HDG solution $(k=4)$ of Poiseuille flow along a channel of square cross-section.

Table 1. Poiseuille flow along a channel with square cross-section solved by the HDG. Itr denotes the number of iteration steps to satisfy the residual less than $10^{-5}$, and $t_{\mathrm{c}}$ is the CPU time.

\begin{tabular}{cccccccc|ccc}
\hline \multirow{2}{*}{$k$} & \multirow{2}{*}{$M_{\mathrm{el}}$} & \multicolumn{2}{c}{$K n=0.018$} & \multicolumn{4}{c}{$K n=0.089$} \\
\cline { 2 - 11 } & $L_{1}$ error & Itr & $t_{\mathrm{c}},[\mathrm{s}]$ & $L_{1}$ error & Itr & $t_{\mathrm{c}},[\mathrm{s}]$ & $L_{1}$ error & Itr & $t_{\mathrm{c}},[\mathrm{s}]$ \\
\hline \multirow{4}{*}{1} & 8 & $3.15 \times 10^{-1}$ & 1283 & 111.6 & $9.92 \times 10^{-2}$ & 131 & 10.8 & $1.05 \times 10^{-2}$ & 15 & 1.3 \\
& 18 & $1.49 \times 10^{-1}$ & 1468 & 284.5 & $4.28 \times 10^{-2}$ & 137 & 24.6 & $4.18 \times 10^{-3}$ & 15 & 2.6 \\
& 32 & $8.34 \times 10^{-2}$ & 1539 & 529.4 & $2.32 \times 10^{-2}$ & 139 & 45.0 & $2.20 \times 10^{-3}$ & 15 & 4.6 \\
& 50 & $5.26 \times 10^{-2}$ & 1570 & 852.0 & $1.46 \times 10^{-2}$ & 140 & 70.8 & $1.37 \times 10^{-3}$ & 15 & 9.3 \\
\hline \multirow{4}{*}{2} & 8 & $2.46 \times 10^{-2}$ & 1595 & 214.3 & $6.62 \times 10^{-3}$ & 141 & 18.0 & $4.04 \times 10^{-4}$ & 15 & 1.9 \\
& 18 & $9.99 \times 10^{-3}$ & 1612 & 498.4 & $3.65 \times 10^{-3}$ & 141 & 39.8 & $3.98 \times 10^{-4}$ & 15 & 4.2 \\
& 32 & $7.16 \times 10^{-3}$ & 1615 & 887.0 & $2.93 \times 10^{-3}$ & 141 & 71.5 & $3.65 \times 10^{-4}$ & 15 & 8.0 \\
& 50 & $6.29 \times 10^{-3}$ & 1615 & 1380.8 & $2.64 \times 10^{-3}$ & 141 & 112.5 & $3.47 \times 10^{-4}$ & 15 & 12.2 \\
\hline \multirow{4}{*}{3} & 8 & $6.54 \times 10^{-3}$ & 1615 & 305.5 & $2.84 \times 10^{-3}$ & 141 & 24.2 & $3.71 \times 10^{-4}$ & 15 & 3.6 \\
& 18 & $5.78 \times 10^{-3}$ & 1615 & 696.3 & $2.50 \times 10^{-3}$ & 141 & 55.6 & $3.43 \times 10^{-4}$ & 15 & 9.2 \\
& 32 & $5.59 \times 10^{-3}$ & 1615 & 1248.1 & $2.39 \times 10^{-3}$ & 141 & 97.8 & $3.29 \times 10^{-4}$ & 15 & 10.6 \\
& 50 & $6.75 \times 10^{-3}$ & 1615 & 1939.9 & $2.33 \times 10^{-3}$ & 141 & 160.7 & $3.24 \times 10^{-4}$ & 15 & 17.5 \\
\hline \multirow{4}{*}{4} & 2 & $6.75 \times 10^{-3}$ & 1615 & 122.7 & $2.77 \times 10^{-3}$ & 141 & 10.0 & $6.90 \times 10^{-5}$ & 15 & 1.1 \\
& 8 & $5.67 \times 10^{-3}$ & 1615 & 490.0 & $2.44 \times 10^{-3}$ & 141 & 37.7 & $3.33 \times 10^{-4}$ & 15 & 4.0 \\
& 18 & $5.48 \times 10^{-3}$ & 1615 & 1125.0 & $2.33 \times 10^{-3}$ & 141 & 89.0 & $3.24 \times 10^{-4}$ & 15 & 9.4 \\
& 32 & $5.37 \times 10^{-3}$ & 1616 & 2008.3 & $2.29 \times 10^{-3}$ & 141 & 152.4 & $3.20 \times 10^{-4}$ & 15 & 18.2 \\
\hline
\end{tabular}

shown in Fig. 1(b)-1(d), respectively. The numbers of triangles in these cases are 50, 32 and 18, respectively. The maximum velocity emerges in the center of the flow field. As Knudsen number (degree of rarefaction) increases, the maximum velocity reduces while the slip velocity in the vicinity of solid surfaces increases.

To investigate performance of the proposed scheme, the $L_{1}$ error of the dimensionless mass flow rate (MFR, $M=\int u_{3} \mathrm{~d} \Delta$ ), the number of iterative steps and the CPU time are listed in Table 1, for various numbers of triangles and degrees of approximation polynomials. The solutions from the discrete unified gas kinetic scheme, which have been verified for a wide range of rarefactions (Wang et al. 2018), are used as reference for error estimation. Note that, in some cases, the error does not reduce monotonically with increasing number of triangles. This is due to the fact that the reference data are also numerical. As expected, on the same spatial grid, the HDG solutions of higherorder accuracy are obtained using higher-order approximating polynomials. Thereby, to achieve the same order of accuracy, the solvers with higher-order polynomials require fewer triangles. On the other hand, for each rarefaction level, the solvers with different degree of polynomials require almost the same number of iterations to obtain steadystate solutions. Since fewer triangles are needed, the higher order the solver, the less the CPU time. For instance, at $K n=0.018$, the CPU time to obtain solution of $\sim 0.7 \%$ error for $k=4$ is about $40 \%$ and $14 \%$ of that for the solvers with $k=3$ and $k=2$, respectively. It is also interesting to compare the performances of the HDG and the original DG methods. The comparison is for the flow at $K n=0.089$. In DG scheme, the same semi-implicit iterative scheme is applied. Unlike HDG, it doesn't resolve $\hat{h}^{j}$. Instead, a global linear system is directly built up for all the DoF of field variables $h^{j}$. The numerical flux is modeled through the upwind scheme, see Eq. (11). The number 
Table 2. Comparison of the implicit HDG and DG. Itr denotes the number of iteration steps to satisfy the residual less than $10^{-5}, N_{\text {dof }}$ denotes the number of globally coupled DoF, and $t_{\mathrm{c}}$ is the CPU time. Note that Itr and $L_{1}$ in both methods are exactly the same.

\begin{tabular}{cccccccccc}
\hline \multirow{2}{*}{$k$} & \multirow{2}{*}{$M_{\mathrm{el}}$} & \multicolumn{7}{c}{ HDG } & \multicolumn{5}{c}{ DG } \\
\cline { 3 - 10 } & 8 & 32 & $9.92 \times 10^{-2}$ & 131 & 10.8 & 24 & $9.92 \times 10^{-2}$ & 131 & 4.9 \\
& 32 & 112 & $2.32 \times 10^{-2}$ & 139 & 45.0 & 96 & $2.32 \times 10^{-2}$ & 139 & 21.4 \\
1 & 72 & 240 & $1.02 \times 10^{-2}$ & 141 & 160.0 & 216 & $1.02 \times 10^{-2}$ & 141 & 54.5 \\
& 128 & 416 & $6.22 \times 10^{-3}$ & 141 & 180.2 & 384 & $6.22 \times 10^{-3}$ & 141 & 116.6 \\
\hline & 8 & 48 & $6.62 \times 10^{-3}$ & 141 & 18.0 & 48 & $6.62 \times 10^{-3}$ & 141 & 11.6 \\
& 32 & 168 & $2.93 \times 10^{-3}$ & 141 & 71.5 & 192 & $2.93 \times 10^{-3}$ & 141 & 52.5 \\
2 & 72 & 360 & $2.51 \times 10^{-3}$ & 141 & 169.7 & 432 & $2.51 \times 10^{-3}$ & 141 & 140.3 \\
& 128 & 624 & $2.39 \times 10^{-3}$ & 141 & 316.1 & 768 & $2.39 \times 10^{-3}$ & 141 & 276.6 \\
\hline \multirow{4}{*}{3} & 8 & 64 & $2.84 \times 10^{-3}$ & 141 & 24.2 & 80 & $2.84 \times 10^{-3}$ & 141 & 23.2 \\
& 32 & 224 & $2.39 \times 10^{-3}$ & 141 & 97.8 & 320 & $2.39 \times 10^{-3}$ & 141 & 110.2 \\
& 72 & 480 & $2.30 \times 10^{-3}$ & 141 & 240.9 & 720 & $2.30 \times 10^{-3}$ & 141 & 284.5 \\
& 128 & 832 & $2.28 \times 10^{-3}$ & 141 & 465.8 & 1280 & $2.28 \times 10^{-3}$ & 141 & 523.5 \\
\hline & 8 & 80 & $2.44 \times 10^{-3}$ & 141 & 37.7 & 120 & $2.44 \times 10^{-3}$ & 141 & 44.0 \\
4 & 32 & 280 & $2.29 \times 10^{-3}$ & 141 & 152.4 & 480 & $2.29 \times 10^{-3}$ & 141 & 216.1 \\
& 72 & 600 & $2.27 \times 10^{-3}$ & 141 & 381.2 & 1080 & $2.27 \times 10^{-3}$ & 141 & 545.0 \\
& 128 & 1040 & $2.26 \times 10^{-3}$ & 141 & 735.1 & 1920 & $2.26 \times 10^{-3}$ & 141 & 893.8 \\
\hline
\end{tabular}

of DoF $N_{\text {dof }}$, the $L_{1}$ error in MFR, the number of iterative steps, and the CPU time are listed in Table 2 for both approaches. It shows that HDG and DG with the same order approximating polynomial yield the same solution (MFRs have at least 7 same significant digits) on the same mesh, and consume the same number of iterative steps. For HDG, the numbers of globally coupled DoF is $N_{\text {dof }}=M_{\mathrm{v}} M_{\mathrm{fc}}(k+1)$, while for $\mathrm{DG}, N_{\mathrm{dof}}=M_{\mathrm{v}} M_{\mathrm{el}}(k+1)(k+2) / 2$. Here, $M_{\mathrm{fc}}$ is around 1.7 times of $M_{\mathrm{el}}$. It is found that $N_{\text {dof }}$ in HDG is smaller than the one in DG when $k \geq 2$. The higher order and more triangles, the more significant this difference will be. As a consequence, the HDG method cost less CPU time than the DG method when $k>2$. For example, HDG with $k=4$ on 128 triangles can save $30 \%$ CPU time, compared to the original DG method. Note that $N_{\text {dof }}$ of HDG with $k=2$ is smaller than that of DG. However, it is not more efficient, since extra effort is required to recover field solution from trace solution in the HDG scheme.

Finally, we show the solutions of HDG to the nonlinear Boltzmann equation for the square cavity flow driven by its top lid of horizontal velocity $U=0.148$ at $K n=1$ and thermal flow induced by non-uniform temperature field between two coaxial elliptic cylinders at $K n=0.5$. The temperature contours from HDG (background) and the direct simulation Monte Carlo (DSMC) method (red dashed lines) (John, Gu, and Emerson 2010), as well as the streamlines for cavity flow are shown in Fig. 22(a). The HDG solution, obtained with $k=4$ on 72 uniform triangles using $108 \times 108 \times 24$ discrete velocities, agrees well with the DSMC result. The geometry and solutions of gas temperature and streamlines for the thermal flow are illustrated in Fig. 2(b)-(c), where the cold cylinder is encompassed in a hot chamber. Only the upper right quarter of the domain is shown due to symmetry. Unlike the continuum flow where the bulk velocity is zero, at rarefied conditions, temperature inhomogeneity induces anisotropic momentum transfer that produces gas flow with two vortexes in the field. Temperature distribution from HDG $(k=2$, on 142 unstructured triangles and $96 \times 96 \times 24$ discrete velocities) is closed to the DSMC results (Aoki, Sone, and Waniguchi 1998), which shows capacity of the developed solver to deal with more complex geometry. The full diffuse wall condition is used in the two cases. 


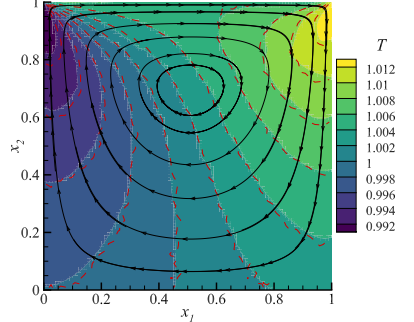

(a) Cavity flow driven by top lid with $U=0.148$ at $K n=1$

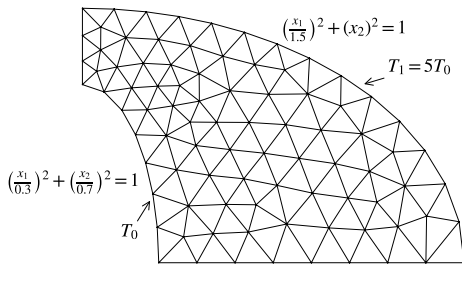

(b) Configuration for flow induced by temperature inhomogeneity

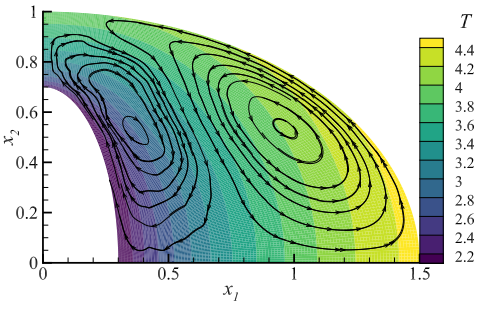

(c) Thermal flow induced by temperature inhomogeneity at $K n=0.5$

Figure 2. Examples of HDG solutions to the nonlinear Boltzmann equation.

\section{Conclusions}

In summary, we have developed a high-order HDG solver for the solution of gas kinetic equations on arbitrary triangular mesh. The velocity space is first discretized using a quadrature rule. Then, the discrete molecular VDFs and their traces are approximated in piecewise polynomial space of degree up to 4 on the spatial mesh and the mesh skeleton, respectively. Based on the upwind scheme, a numerical flux has been designed to evaluate the convection between adjacent cells. By imposing the continuity of the normal flux, global system is setup in terms of the unknown traces only. Once the traces are resolved, the VDFs are updated in an element-by-element fashion. The boundary condition has been implemented in the same framework as the calculation of flux on interfaces. Finally, an implicit iterative scheme is employed to obtain the steady-state solution. Performance analysis shows that, to obtain the results with the same order of accuracy, the scheme with higher-order approximating polynomial requires fewer triangles in spatial discretization. As a result, the computational time and memory consumption are reduced. Furthermore, compared to the original DG scheme, the HDG solver is more efficient when the degree of approximating polynomial is larger than 2 , since HDG has fewer number of globally coupled degrees of freedom.

\section{References}

Aoki, K., Y. Sone, and Y. Waniguchi. 1998. "A rarefied gas flow induced by a temperature field: Numerical analysis of the flow between two coaxial elliptic cylinders with different uniform temperatures." Computers 83 Mathematics with Applications 35 (1): 15 - 28.

Bhatnagar, P. L., E. P. Gross, and M. Krook. 1954. "A Model for Collision Processes in Gases. I. Small Amplitude Processes in Charged and Neutral One-Component Systems." Physical Review 94: 511-525.

Cercignani, C. 1988. The Boltzmann Equation and its Applications. Springer-Verlag, New York.

Chapman, S., and T.G. Cowling. 1970. The Mathematical Theory of Non-uniform Gases. 3rd ed. Cambridge University press, New York.

Cockburn, B., J. Gopalakrishnan, and R. Lazarov. 2009. "Unified Hybridization of Discontinuous Galerkin, Mixed, and Continuous Galerkin Methods for Second Order Elliptic Problems." SIAM Journal on Numerical Analysis 47 (2): 1319-1365.

Cockburn, B., and C.-W. Shu. 2001. "Runge-Kutta Discontinuous Galerkin Methods for Convection-Dominated Problems." Journal of Scientific Computing 16 (3): 173-261.

Dimarco, G., and L. Pareschi. 2014. "Numerical methods for kinetic equations." Acta Numerica 23: 369-520.

Evans, B., K. Morgan, and O. Hassan. 2011. "A discontinuous finite element solution of the 
Boltzmann kinetic equation in collisionless and BGK forms for macroscopic gas flows." Applied Mathematical Modelling 35 (3): 996 - 1015.

Giorgiani, G., S. Fernández-Méndez, and A. Huerta. 2013. "Hybridizable discontinuous Galerkin p-adaptivity for wave propagation problems." International Journal for Numerical Methods in Fluids 72 (12): 1244-1262.

Gobbert, M. K., S. G. Webster, and T. S. Cale. 2007. "A Galerkin Method for the Simulation of the Transient 2-D/2-D and 3-D/3-D Linear Boltzmann Equation." Journal of Scientific Computing 30 (2): 237-273.

Huerta, A., A. Angeloski, X. Roca, and J. Peraire. 2013. "Efficiency of high-order elements for continuous and discontinuous Galerkin methods." International Journal for Numerical Methods in Engineering 96 (9): 529-560.

John, Benzi, Xiao-Jun Gu, and David R. Emerson. 2010. "Investigation of Heat and Mass Transfer in a Lid-Driven Cavity Under Nonequilibrium Flow Conditions." Numerical Heat Transfer, Part B: Fundamentals 58 (5): 287-303.

Kirby, R. M., S. J. Sherwin, and B. Cockburn. 2012. "To CG or to HDG: A Comparative Study." Journal of Scientific Computing 51 (1): 183-212.

Kitzler, G., and J. Schöberl. 2015. "A high order space-momentum discontinuous Galerkin method for the Boltzmann equation." Computers 8 Mathematics with Applications 70 (7): 1539 - 1554.

Kubatko, E. J., C. Dawson, and J. J. Westerink. 2008. "Time step restrictions for Runge-Kutta discontinuous Galerkin methods on triangular grids." Journal of Computational Physics 227 (23): $9697-9710$.

Mieussens, L. 2014. "A survey of deterministic solvers for rarefied flows (Invited)." AIP Conference Proceedings 1628 (1): 943-951.

Nguyen, N.C., J. Peraire, and B. Cockburn. 2010. "A hybridizable discontinuous Galerkin method for Stokes flow." Computer Methods in Applied Mechanics and Engineering 199 (9): $582-597$.

Peraire, J., N. Nguyen, and B. Cockburn. 2010. "A Hybridizable Discontinuous Galerkin Method for the Compressible Euler and Navier-Stokes Equations." In 48th AIAA Aerosp. Sci. Meet. Incl. New Horizons Forum Aerosp. Expo., 1-11.

Reed, W. H., and T. R. Hill. 1973. Triangular Mesh Methods for the Neutron Transport Equation. Technical Report 836.

Schenk, O., and K. Gärtner. 2004. "Solving unsymmetric sparse systems of linear equations with PARDISO." Future Generation Computer Systems 20 (3): 475 - 487.

$\mathrm{Su}$, W., A. A. Alexeenko, and G. Cai. 2015. "A parallel Runge-Kutta discontinuous Galerkin solver for rarefied gas flows based on 2D Boltzmann kinetic equations." Computers Es Fluids 109: $123-136$.

Su, W., S. Lindsay, H. Liu, and L. Wu. 2017. "Comparative study of the discrete velocity and lattice Boltzmann methods for rarefied gas flows through irregular channels." Physics Review E 96: 023309.

Wang, P., M. T. Ho, L. Wu, Z. Guo, and Y. Zhang. 2018. "A comparative study of discrete velocity methods for low-speed rarefied gas flows." Computers \& Fluids 161: 33 - 46.

Wu, L., C. White, T. J. Scanlon, J. M. Reese, and Y. Zhang. 2013. "Deterministic numerical solutions of the Boltzmann equation using the fast spectral method." Journal of Computational Physics 250: $27-52$.

\section{Appendix}

Unknowns are approximated by the nodal shape functions $N_{l}$ in each triangle $\Delta_{i}$ or by $\hat{N}_{l}$ on each face $\Gamma_{c}: h_{i}^{j}=\sum_{l=1}^{K_{\mathrm{el}}} N_{i}^{l} H_{i, l}^{j}, \hat{h}_{c}^{j}=\sum_{l=1}^{K_{\mathrm{fc}}} \hat{N}_{c}^{l} \hat{H}_{c, l}^{j}$, where $K_{\mathrm{el}}=(k+1)(k+2) / 2$ and $K_{\mathrm{fc}}=k+1$ are the numbers of DoF. Denote $\mathbf{H}^{i, j}$ as the vector of nodal value of 
$h^{j}$ on each $\Delta_{i}, \hat{\mathbf{H}}^{i, j}$ as the vector summing all the nodal value of $\hat{h}^{j}$ on the three faces of $\Delta_{i}$, and $\hat{\mathbf{H}}^{c, j}$ as the vector of nodal value of $\hat{h}^{j}$ on each $\Gamma_{c}$, the local and global problems can be rewritten in the matrix form

$$
\mathbf{H}^{i, j}=\left[\mathbf{A}^{i, j}\right]^{-1} \mathbf{S}^{i, j}+\left[\mathbf{A}^{i, j}\right]^{-1} \hat{\mathbf{A}}^{i, j} \hat{\mathbf{H}}^{i, j}
$$

and

$$
\begin{aligned}
\hat{\mathbf{B}}^{c, j} \hat{\mathbf{H}}^{c, j}= & \mathbf{B}^{\eta(c,+), j} \mathbf{H}^{\eta(c,+), j}+\mathbf{B}^{\eta(c,-), j} \mathbf{H}^{\eta(c,-), j}, \quad \text { on } \Gamma \backslash \partial \Omega, \\
& \hat{\mathbf{B}}^{c, j} \hat{\mathbf{H}}^{c, j}=\mathbf{B}^{\eta(c,+), j} \mathbf{H}^{\eta(c,+), j}+\hat{\mathbf{S}}^{c, j}, \quad \text { on } \Gamma \cap \partial \Omega,
\end{aligned}
$$

where

$$
\begin{array}{r}
\mathbf{A}_{m l}^{i, j}=\frac{\sqrt{\pi}}{2 K n}\left(N_{i}^{m}, N_{i}^{l}\right)_{\Delta_{i}}+\sum_{e=1}^{3}\left|\boldsymbol{v}^{j} \cdot \boldsymbol{n}\right|\left\langle N_{i}^{m}, N_{i}^{l}\right\rangle_{\partial \Delta_{i}^{e}}-\left(\boldsymbol{v}^{j} \cdot \nabla N_{i}^{m}, N_{i}^{l}\right)_{\Delta_{i}}, \\
\hat{\mathbf{A}}_{m l}^{i, j, e}=\left(\left|\boldsymbol{v}^{j} \cdot \boldsymbol{n}\right|-\boldsymbol{v}^{j} \cdot \boldsymbol{n}\right)\left\langle N_{i}^{m}, \hat{N}_{\sigma(i, e)}^{l}\right\rangle_{\partial \Delta_{i}^{e}}, \mathbf{S}_{m}^{i, j}=\left(N_{i}^{m}, s^{j}\right)_{\Delta_{i}} \\
\hat{\mathbf{B}}_{m l}^{c, j}=\left\langle\hat{N}_{c}^{m}, \hat{N}_{c}^{l}\right\rangle_{\Gamma_{c}}, \mathbf{B}_{m l}^{\eta(c, \pm), j}=\frac{1}{2}\left\langle\hat{N}_{c}^{m}, N_{\eta(c, \pm)}^{l}\right\rangle_{\Gamma_{c}}, \hat{\mathbf{S}}_{m}^{c, j}=\frac{1}{2}\left\langle\hat{N}_{c}^{m}, g^{j}\right\rangle_{\Gamma_{c}}
\end{array}
$$

By eliminating the unknowns $\mathbf{H}^{i, j}$ with Eq. A.1) and assembling the equations of global problem over all faces, the global problem becomes $\mathbb{K}^{j} \hat{\mathbf{H}}^{j}=\mathbb{R}^{j}$ with

$$
\begin{aligned}
\mathbb{K}^{j} & =\bigwedge_{c=1}^{M_{\mathrm{fc}}}\left\{\hat{\mathbf{B}}^{c, j}-\mathbf{B}^{\eta(c, \pm), j}\left[\mathbf{A}^{\eta(c, \pm), j}\right]^{-1} \hat{\mathbf{A}}^{\eta(c, \pm), j}\right\}, \\
\mathbb{R}^{j} & =\bigwedge_{c=1}^{M_{\mathrm{fc}}}\left\{\mathbf{B}^{\eta(c, \pm), j}\left[\mathbf{A}^{\eta(c, \pm), j}\right]^{-1} \mathbf{S}^{\eta(c, \pm), j}+\hat{\mathbf{S}}^{c, j}\right\} .
\end{aligned}
$$

Here, $\mathrm{A}$ is the conventional assembly operator.

When the nonlinear Boltzmann equation is solved, the unkowns are the full VDF and its trace, which are approximated as $f_{i}^{j}=\sum_{l=1}^{K_{\mathrm{el}}} N_{i}^{l} F_{i, l}^{j}, \hat{f}_{c}^{j}=\sum_{l=1}^{K_{\mathrm{fc}}} \hat{N}_{c}^{l} \hat{F}_{c, l}^{j}$ with $F$ and $\hat{F}$ being the corresponding nodal values. Then the terms $\sqrt{\pi}\left(N_{i}^{m}, N_{i}^{l}\right)_{\Delta_{i}} / 2 K n$ and $\left(N_{i}^{m}, s^{j}\right)_{\Delta_{i}}$ in A.3 are replaced by

$$
\sum_{r=1}^{K_{\mathrm{el}}}\left(N_{i}^{m}, N_{i}^{r} N_{i}^{l}\right)_{\Delta_{i}} \iint B F_{*, i, r}^{j} \mathrm{~d} \Omega \mathrm{d} \boldsymbol{v}_{*}
$$

and

$$
\sum_{r=1}^{K_{\mathrm{el}}} \sum_{l=1}^{K_{\mathrm{el}}}\left(N_{i}^{m}, N_{i}^{r} N_{i}^{l}\right)_{\Delta_{i}} \iint B F_{*, i, r}^{\prime, j} F_{i, l}^{\prime, j} \mathrm{~d} \Omega \mathrm{d} \boldsymbol{v}_{*}-\boldsymbol{a} \cdot \sum_{l=1}^{K_{\mathrm{el}}}\left(N_{i}^{m}, N_{i}^{l}\right)_{\Delta_{i}} \frac{\partial F_{i, l}^{j}}{\partial \boldsymbol{v}},
$$

respectively. The integral $\iint \cdot \mathrm{d} \Omega \mathrm{d} \boldsymbol{v}_{*}$ and derivative $\partial / \partial \boldsymbol{v}$ are calculated via the fast spectral method. 